\title{
New Results in Controlled Metric Type Spaces
}

\author{
Muhib Abuloha, ${ }^{1}$ Doaa Rizk, ${ }^{2}$ Kamaleldin Abodayeh $\mathbb{D}^{3}{ }^{3}$ Nabil Mlaiki, ${ }^{3}$ \\ and Thabet Abdeljawad $\mathbb{( D}^{3,4,5}$ \\ ${ }^{1}$ Palestine Technical University-Kadoorie, Tulkarm, State of Palestine \\ ${ }^{2}$ Department of Mathematics, Faculty of Science, Al Qassim University, College of Science and Arts, Al-Asyah, Saudi Arabia \\ ${ }^{3}$ Department of Mathematics and General Sciences, Prince Sultan University, P.O. Box 66833, Riyadh 11586, Saudi Arabia \\ ${ }^{4}$ Department of Medical Research, China Medical University, Taichung 40402, Taiwan \\ ${ }^{5}$ Department of Computer Sciences and Information Engineering, Asia University, Taichung, Taiwan
}

Correspondence should be addressed to Kamaleldin Abodayeh; kamal@psu.edu.sa and Thabet Abdeljawad; tabdeljawad@ psu.edu.sa

Received 3 February 2021; Revised 5 March 2021; Accepted 17 March 2021; Published 1 April 2021

Academic Editor: Naeem Saleem

Copyright (c) 2021 Muhib Abuloha et al. This is an open access article distributed under the Creative Commons Attribution License, which permits unrestricted use, distribution, and reproduction in any medium, provided the original work is properly cited.

In this paper, a new class of functions denoted by $\Psi_{v}$ is introduced which we use to prove new interesting fixed point results in controlled metric type spaces. Also, we present examples to illustrate our work.

\section{Introduction}

Fixed point theory is one of the most interesting topics that was introduced by Banach [1] in 1922. Since that time and for the last decades, this area of research has become the inspiration for many researchers in the field of nonlinear analysis and its applications. We refer the reader to check these extensions of the Banach theorem in different metric spaces; for example, in [2], the authors proved the existence of a fixed point for a self-mapping on $S_{b}-$ metric spaces that satisfies a more general contraction.

In 1989, Bakhtin [3] introduced an extension of metric spaces, called $b$-metric spaces, where many interesting fixed point results for some contractive mappings in $b$-metric spaces were studied. Also, in 1993, Czerwik [4] extended the results of $b$-metric spaces. In 2018, Shatanawi et al. [5] introduced the $\alpha-\psi$-contraction on the extended $b$-metric spaces. Recently, many research studies were conducted on b-metric space under different contraction conditions. After that, many authors used $\alpha-\psi$-contraction mapping on different metric spaces (see $[6,7])$. In 2017, Kamran et al. [8] presented a very interesting generalization of the $b$-metric spaces, called extended b-metric spaces. An extension of the extended b-metric spaces, called controlled metric type space, was introduced by Mlaiki et al. [9].

In this paper, we generalize the results of Mehmet [10] and Mukheimer [11] by introducing the $\alpha-\psi$-contractive mapping on controlled metric type spaces.

\section{Preliminaries}

The concept of extended $b$-metric spaces was initiated by Kamran et al. [8] in 2017, and their work generalized many results in the literature (see, for example, [12-16]).

Definition 1 (see [8]). Let $Y$ be an nonempty set and define the mappings $\quad v: Y \times Y \longrightarrow[1, \infty)$ and $\bar{\wp}: Y \times Y \longrightarrow[0, \infty)$, such that $\forall g, h, r \in Y$,

(1) $\bar{\wp}(g, h)=0 \Leftrightarrow g=h$.

(2) $\bar{\wp}(g, h)=\bar{\wp}(h, g)$.

(3) $\bar{\wp}(g, h) \leq v(g, h)[\bar{\wp}(g, r)+\bar{\wp}(r, h)]$.

Then, we say that the pair $(Y, \bar{\wp})$ is an extended $b$-metric space.

A general extension of the $b$-metric spaces was considered by Mlaiki et al. in [9] where the concepts of controlled metric type spaces is defined as follows. 
Definition 2 (see [9]). On a nonempty set $Y$, define the mappings $v: Y \times Y \longrightarrow[1, \infty)$ and $\bar{\wp}: Y \times Y \longrightarrow[0, \infty)$, such that $\forall g, h, r \in Y$, the following conditions hold:

(d1) $\bar{\wp}(g, h)=0$ if and only if $g=h$.

(d2) $\bar{\wp}(g, h)=\bar{\wp}(h, g)$.

(d3) $\bar{\wp}(g, h) \leq v(g, r) \bar{\wp}(g, r)+v(r, h) \bar{\wp}(r, h)$.

Then, the pair $(Y, \bar{\wp})$ is called a controlled metric type space.

To illustrate the above definition, we present the following examples.

Example 1 (see [9]). Choose $Y=\{1,2, \ldots$,$\} . Take \bar{\wp}: Y \times$ $Y \longrightarrow[0, \infty)$ such that

$$
\bar{\wp}(g, h)= \begin{cases}0, & \Leftrightarrow g=h, \\ \frac{1}{g}, & \text { if } g=2 n \text { and } h=2 n+1, \\ \frac{1}{h}, & \text { if } g=2 n+1 \text { and } h=2 n, \\ 1, & \text { otherwise. }\end{cases}
$$

Consider $v: Y \times Y \longrightarrow[1, \infty)$ as

$$
v(g, h)= \begin{cases}g, & \text { if } g=2 n \text { and } h=2 n+1 \\ h, & \text { if } g=2 n+1 \text { and } h=2 n \\ 1, & \text { otherwise }\end{cases}
$$

It is clear that the conditions $(d 1)$ and $(d 2)$ are satisfied. Now, we investigate condition (d3).

Case 1. If $r=g$ or $r=h$, (d3) is satisfied.

Case 2. If $r \neq g$ and $r \neq h$, (d3) holds when $g=h$. Now, we may assume that $g \neq h$. Then, we have $g \neq h \neq r$. It is clear that (d3) holds in all following possible subcases:

(1) $g, r$ are even and $h=2 n+1$.

(2) $g=2 n$ and $h, r$ are odd.

(3) $g, r$ are odd and $h=2 n$.

(4) $g, r$ are even and $h=2 n+1$.

(5) $g, h, r$ are even.

(6) $g, h$ are even and $r=2 n+1$.

(7) $g, h$ are odd and $r=2 n$.

(8) $g, h, r$ are odd.

Thus, $\bar{\wp}$ is a controlled metric type.

Moreover, for $n=2,3, \ldots$, we have

$$
\begin{aligned}
\bar{\wp}(2 n+1,4 n+1)= & 1>\frac{1}{n}=\nu(2 n+1,4 n+1) \\
& \cdot[\bar{\wp}(2 n+1,2 n)+\bar{\wp}(2 n, 4 n+1)] .
\end{aligned}
$$

Therefore, $\bar{\wp}$ is not an extended $b$-metric.
Example 2 (see [9]). Take $Y=\{0,1,2\}$. Consider the function $\bar{\wp}$ given as

$$
\begin{aligned}
& \bar{\wp}(0,0)=\bar{\wp}(1,1)=\bar{\wp}(2,2)=0, \\
& \bar{\wp}(0,1)=\bar{\wp}(1,0)=1, \\
& \bar{\wp}(0,2)=\bar{\wp}(2,0)=\frac{1}{2}, \\
& \bar{\wp}(1,2)=\bar{\wp}(2,1)=\frac{2}{5} .
\end{aligned}
$$
that

Define a symmetric function $v: Y \times Y \longrightarrow[1, \infty)$ such

$$
\begin{aligned}
& \nu(0,0)=\nu(1,1)=\nu(2,2)=v(0,2)=1, \\
& v(1,2)=\frac{5}{4}, \\
& \nu(0,1)=\frac{11}{10} .
\end{aligned}
$$

One can easily verify that $\bar{\wp}$ is a controlled metric type. Since

$$
\bar{\wp}(0,1)=1>\frac{99}{100}=\nu(0,1)[\bar{\wp}(0,2)+\bar{\wp}(2,1)],
$$

$\bar{\wp}$ is not an extended $b$-metric.

The concepts of Cauchy and convergent sequences in controlled metric type spaces are defined as follows.

Definition 3. Let $(Y, \bar{\wp})$ be a controlled metric type space and $\left\{g_{n}\right\}_{n \geq 0}$ be a sequence in $Y$.

(1) The sequence $\left\{g_{n}\right\}$ converges to some $g \in Y$, if $\forall \epsilon>0, \quad \exists N=N(\varepsilon) \in \mathbb{N}$ such that $\bar{\wp}\left(g_{n}, g\right)<\varepsilon \forall n \geq N$. We write $\lim _{n \longrightarrow \infty} g_{n}=g$.

(2) We say that $\left\{g_{n}\right\}$ is Cauchy, if $\forall \varepsilon>0, \exists N=N(\varepsilon) \in \mathbb{N}$ such that $\bar{\wp}\left(g_{m}, g_{n}\right)<\varepsilon \forall m, n \geq N$.

(3) If every Cauchy sequence is convergent, then the space $(Y, \bar{\wp})$ is called complete.

Definition 4 (see [9]). Let $(Y, \bar{\wp})$ be a controlled metric type space. Let $g \in Y$ and $\varepsilon>0$.

(i) The open ball $B(g, \varepsilon)$ is defined as

$$
B(g, \varepsilon)=\{h \in Y, \bar{\wp}(g, h)<\varepsilon\} .
$$

(ii) A self-mapping $\xi$ on $Y$ is said to be continuous at $g \in Y$, if $\forall \varepsilon>0, \exists \delta>0$ such that $\xi(B(g, \delta)) \subseteq B(\xi g, \varepsilon)$.

Remark 1. If for all $g, y$ in $Y, v(g, h)=s \geq 1$, then $(Y, \bar{\wp})$ is a $b$-metric space. Therefore, we conclude that every $b$-metric space is a controlled metric type space. However, the converse is not always true.

Clearly, if a mapping $\xi$ is continuous at $g$ in the controlled metric type space $(Y, \bar{\wp})$, then $g_{n} \longrightarrow g$ implies that $\xi g_{n} \longrightarrow \xi g$ as $n \longrightarrow \infty$. 
Let $\Psi$ denote to the set of all functions $\psi:[0, \infty) \longrightarrow[0, \infty)$ such that

(1) $\psi$ is nondecreasing.

(2) $\sum_{n=1}^{\infty} \psi^{n}(t)<+\infty$ for all $t>0$, where $\psi^{n}$ is the $n$-th iterate of $\psi$.

Now, we recall the following lemma.

Lemma 1 (see [5]). If $\psi \in \Psi$, then $\psi(t)<t$, for all $t \in(0,+\infty)$.

Next, we introduce the following class of functions.

Definition 5 (see [5]). Let $Y$ be a nonempty set and $v$ : $Y \times$ $Y \longrightarrow[1,+\infty)$ be a mapping. A function $\psi:[0, \infty) \longrightarrow[0, \infty)$ is said to be controlled comparison function if $\psi$ satisfies the following conditions:

(1) $\psi$ is nondecreasing.

(2) $\sum_{n=1}^{\infty} \psi^{n}(t) \prod_{i=1}^{n} v\left(g_{i}, g_{m}\right) v\left(g_{n}, g_{n+1}\right)<+\infty$, and $\lim _{n \rightarrow \infty} \psi^{n}(t) v\left(g_{n}, g_{n+1}\right)<\infty$ for any sequence $\left\{g_{n}\right\}_{n=1}^{\infty}$ in $Y$, for all $t>0$ and non-negative integer $m$ where $\psi^{n}$ is the $n$-th iterate of $\psi$.

The set of all controlled comparison functions is denoted by $\Psi_{v}$ which is an extension of $b$-comparison functions of Berinde.

Note that if $\psi \in \Psi_{v}$, then we have $\sum_{n=1}^{\infty} \psi^{n}(t)<\infty$, since $\psi^{n}(t) \prod_{i=1}^{n} v\left(g_{i}, g_{m}\right) \geq \psi^{n}(t)$, for all $t>0$. Hence, by Lemma 1 , we have $\psi(t)<t$.

To show that the family $\Psi_{v}$ is a nonempty set, we present the following examples.

Example 3. Consider the controlled b-metric space $(Y, \bar{\wp})$ which was defined in Example 2. Define the mapping $\psi(t)=(k t / 2)$, where $k<1$. Note that $\nu(g, h) \leq 2$. Then, we have $\psi^{n}(t) \prod_{i=1}^{n} v\left(g_{i}, g_{m}\right) v\left(g_{n}, g_{n+1}\right) \leq\left(k^{n} t / 2^{n}\right) \cdot 2^{n+1}=2 k^{n} t$. Therefore,

$\sum_{n=1}^{\infty} \psi^{n}(t) \prod_{i=1}^{n} v\left(g_{i}, g_{m}\right) v\left(g_{n}, g_{n+1}\right) \leq \sum_{n=1}^{\infty} 2 k^{n} t<\infty$. Similarly, it is not difficult to see that $\lim _{n \longrightarrow \infty} \psi^{n}(t) v\left(g_{n}, g_{n+1}\right)<\infty$.

\section{Main Result}

First, we define the $\alpha$ - $\psi$-contractive self-mapping in controlled metric type spaces.

Definition 6. Let $\xi$ be a self-mapping on a complete controlled metric type space $(Y, \bar{\wp})$. We say that $\xi$ is $\alpha-\psi$-contractive mapping if there exists a function $\alpha: Y^{2} \longrightarrow[0, \infty)$ and $\psi \in \Psi_{\nu}$ such that for all $g, y \in Y$, we have

$$
\alpha(g, h) \bar{\wp}(\xi g, \xi h) \leq \psi(\bar{\wp}(g, h)) .
$$

Definition 7 (see [17]). Let $(Y, \bar{\wp})$ be a controlled metric type space. A mapping $\xi: Y \longrightarrow Y$ is said to be an $\alpha$-admissible if the following condition holds: if $\forall g, y \in Y$, with $\alpha(g, h) \geq 1$, then $\alpha(\xi g, \xi h) \geq 1$.

Now, we prove our first result.

Theorem 1. Let $(Y, \bar{\wp})$ be a complete controlled metric type space and $\xi: Y \longrightarrow Y$ be an $\alpha-\psi$ contractive mapping for some $\psi \in \Psi_{v}$. Assume that

(A) $\xi$ is $\alpha$-admissible.

(B) There exists $g_{0} \in Y$ such that $\alpha\left(g_{0}, \xi g_{0}\right) \geq 1$.

(C) $\xi$ is continuous.

Then, $\xi$ has a fixed point. Moreover, if for any two fixed points of $\xi$ in $Y$ say $a, b$ we have $\alpha(a, b) \geq 1$, then $\xi$ has $a$ unique fixed point in $Y$.

Proof. Take $g_{0}$ to be the point in Condition (2) in our theorem. Define the sequence $\left\{g_{n}\right\}_{n \geq 0}$ by $\xi g_{n}=\xi^{n} g_{0}$.

First of all, note if there exists $n$ such that $g_{n}=g_{n+1}$, then we are done and $g_{n}$ is the fixed point of $\xi$.

So, we may assume that $g_{n} \neq g_{n+1}$ for all $n \geq 0$. Also, we know from the hypothesis of our theorem that $\alpha\left(g_{0}, \xi g_{0}\right) \geq 1, \alpha\left(g_{0}, g_{1}\right) \geq 1$, and using the fact that $\xi$ is $\alpha-$ admissible, we can easily deduce that for all $n \geq 0$, $\alpha\left(g_{n}, g_{n+1}\right) \geq 1$

Now, using the fact that $\xi$ is an $\alpha-\psi$ contractive mapping, we deduce that

$$
\begin{aligned}
\bar{\wp}\left(g_{n}, g_{n+1}\right) & \leq \alpha\left(g_{n-1}, g_{n}\right) \bar{\wp}\left(g_{n}, g_{n+1}\right) \\
& =\alpha\left(g_{n-1}, g_{n}\right) \bar{\wp}\left(\xi g_{n-1}, \xi g_{n}\right) \\
& \leq \psi\left(\bar{\wp}\left(g_{n-1}, g_{n}\right)\right) \\
& \cdot \\
& \cdot \\
& \cdot \\
& \leq \psi^{n}\left(\bar{\wp}\left(g_{0}, g_{1}\right)\right) .
\end{aligned}
$$

Hence, $\forall m, n \in N$ with $m>n$, we have 


$$
\begin{aligned}
& \bar{\wp}\left(g_{n}, g_{m}\right) \leq \nu\left(g_{n}, g_{n+1}\right) \bar{\wp}\left(g_{n}, g_{n+1}\right)+\nu\left(g_{n+1}, g_{m}\right) \bar{\wp}\left(g_{n+1}, g_{m}\right) \\
& \leq v\left(g_{n}, g_{n+1}\right) \bar{\wp}\left(g_{n}, g_{n+1}\right)+v\left(g_{n+1}, g_{m}\right) \nu\left(g_{n+1}, g_{n+2}\right) \bar{\varnothing}\left(g_{n+1}, g_{n+2}\right) \\
& +v\left(g_{n+1}, g_{m}\right) v\left(g_{n+2}, g_{m}\right) \bar{\wp}\left(g_{n+2}, g_{m}\right) \\
& \leq v\left(g_{n}, g_{n+1}\right) \bar{\gamma}\left(g_{n}, g_{n+1}\right)+v\left(g_{n+1}, g_{m}\right) \nu\left(g_{n+1}, g_{n+2}\right) \bar{\gamma}\left(g_{n+1}, g_{n+2}\right) \\
& +v\left(g_{n+1}, g_{m}\right) v\left(g_{n+2}, g_{m}\right) v\left(g_{n+2}, g_{n+3}\right) \bar{\wp}\left(g_{n+2}, g_{n+3}\right) \\
& +v\left(g_{n+1}, g_{m}\right) v\left(g_{n+2}, g_{m}\right) \nu\left(g_{n+3}, g_{m}\right) \bar{\wp}\left(g_{n+3}, g_{m}\right) \\
& \leq \nu\left(g_{n}, g_{n+1}\right) \psi^{n}\left(\bar{\wp}\left(g_{0}, g_{1}\right)\right) \\
& +v\left(g_{n+1}, g_{n+2}\right) \nu\left(g_{n+1}, g_{m}\right) \psi^{n+1}\left(\bar{\wp}\left(g_{0}, g_{1}\right)\right) \\
& +v\left(g_{n+1}, g_{m}\right) v\left(g_{n+2}, g_{m}\right) v\left(g_{n+2}, g_{n+3}\right) \psi^{n+2}\left(\bar{\wp}\left(g_{0}, g_{1}\right)\right) \\
& +v\left(g_{n+1}, g_{m}\right) \nu\left(g_{n+2}, g_{m}\right) \nu\left(g_{n+3}, g_{m}\right) \ldots \nu\left(g_{m-2}, g_{m-1}\right) \psi^{m-1}\left(\bar{\wp}\left(g_{0}, g_{1}\right)\right) \\
& =\nu\left(g_{n}, g_{n+1}\right) \psi^{n}\left(\bar{\wp}\left(g_{0}, g_{1}\right)\right)+\sum_{j=n+1}^{m-2} \psi^{j}\left(\bar{\wp}\left(g_{0}, g_{1}\right)\right) \prod_{i=n+1}^{j} v\left(g_{i}, g_{m}\right) v\left(g_{j}, g_{j+1}\right) \\
& =v\left(g_{n}, g_{n+1}\right) \psi^{n}\left(\bar{\wp}\left(g_{0}, g_{1}\right)\right)+\sum_{j=1}^{m-2} \psi^{j}\left(\bar{\wp}\left(g_{0}, g_{1}\right)\right) \prod_{i=1}^{j} v\left(g_{i}, g_{m}\right) v\left(g_{j}, g_{j+1}\right) \\
& -\sum_{j=1}^{n} \psi^{j}\left(\bar{\wp}\left(g_{0}, g_{1}\right)\right) \prod_{i=1}^{j} v\left(g_{i}, g_{m}\right) \nu\left(g_{j}, g_{j+1}\right) \\
& =S_{m-2}-S_{n}
\end{aligned}
$$

where

$$
S_{m}=\sum_{j=1}^{m} \psi^{j}\left(\bar{\wp}\left(g_{0}, g_{1}\right)\right) \prod_{i=1}^{j} \nu\left(g_{i}, g_{m}\right) \nu\left(g_{j}, g_{j+1}\right) \text {, }
$$

and since $\psi \in \Psi_{\nu}$, we deduce that $\lim _{n, m \rightarrow \infty}\left[S_{m-2}, S_{n}\right]=0$ and

$$
\lim _{n \longrightarrow \infty} \nu\left(g_{n}, g_{n+1}\right) \psi^{n}\left(\bar{\wp}\left(g_{0}, g_{1}\right)\right)<\infty
$$

Thus, the sequence $\left\{g_{n}\right\}_{n \geq 0}$ is a Cauchy sequence. The completeness of the controlled metric type space $(g, \bar{\wp})$ implies that $\left\{g_{n}\right\}$ converges to some $g \in Y$.

Also, note that

$$
\begin{aligned}
\bar{\wp}(g, \xi g) & \leq v\left(g, g_{n+1}\right) \bar{\wp}\left(g, g_{n+1}\right)+v\left(g_{n+1}, \xi g\right) \bar{\wp}\left(g_{n+1}, \xi g\right) \\
& =v\left(g, g_{n+1}\right) \bar{\wp}\left(g, g_{n+1}\right)+v\left(g_{n+1}, \xi g\right) \bar{\wp}\left(\xi g_{n}, \xi g\right) .
\end{aligned}
$$

Taking the limit in above inequality and since $\lim _{n \longrightarrow \infty} \bar{\gamma}\left(g, g_{n+1}\right)=0$ and using the fact that $\xi$ is continuous, we conclude that $\bar{\wp}(g, \xi g)=0$; that is, $\xi g=g$. Thus, $\xi$ has a fixed point as desired. Now, assume that $\xi$ has two fixed points $a, b$ such that $\alpha(a, b) \geq 1$. Hence, using the fact that $\xi$ is an $\alpha-\psi$ contractive mapping and $\xi$ is $\alpha$-admissible, we obtain

$$
\begin{aligned}
\bar{\wp}(a, b) & =\bar{\wp}(\xi a, \xi b) \\
& \leq \alpha(a, b) \bar{\wp}(\xi a, \xi b) \\
& \leq \psi(\bar{\wp}(a, b)) \\
& \vdots \\
& \leq \psi^{n}(\bar{\wp}(a, b)) .
\end{aligned}
$$

Since $\psi \in \Psi_{v}$, taking the limit in the above inequalities, we deduce that $\bar{\wp}(a, b)=0$ which implies that $a=b$. Thus, $\xi$ has a unique fixed point.

Next, we present the following example as an application of Theorem 1 .

Example 4. Let $(Y, \bar{\wp})$ be the controlled metric type space that was defined in Example 2. Define the function $\alpha: Y \times$ $Y \longrightarrow(-\infty, \infty)$ such that

$$
\alpha(g, h)= \begin{cases}1, & \text { if }(g, h)=(1,1), \\ \frac{1}{20}, & \text { if }(g, h) \neq(1,1) .\end{cases}
$$

Define the self-mapping $\xi$ on $Y=\{0,1,2\}$ by $\xi(1)=1, \xi(0)=2, \xi(2)=1$ and the function $\psi(t)=(1 / 8) t$.

We want to verify that $\xi$ satisfies the conditions of Theorem 1. It is clear that $\xi$ is continuous for $g_{0}=1$. We have $\alpha(1, \xi(1))=\alpha(1,1)=1 \geq 1$. So, $\xi$ is $\alpha$-admissible. Now, 
we verify that $\xi$ is $\alpha-\psi$-contractive mapping. Note that $\alpha(g, h)=\alpha(h, g)$.

(i) $\alpha(g, g) \bar{\gamma}(\xi g, \xi g)=0 \leq \psi(\bar{\wp}(g, g))=\psi(0)=0$, for all $g \in Y$.

(ii) $\alpha(1,2) \bar{\wp}(\xi 1, \xi 2)=(1 / 20) \bar{\wp}(1,1)=0 \leq \psi(\bar{\wp}(1,2))=$ $\psi(2 / 5)=(1 / 20)$.

(iii) $\alpha(1,0) \bar{\wp}(\xi 1, \xi 0)=(1 / 20) \bar{\wp}(1,2)=(1 / 50) \leq \psi$ $(\bar{\wp}(1,0))=\psi(1)=(1 / 8)$.

(iv) $\alpha(2,0) \bar{\wp}(\xi 2, \xi 0)=(1 / 20) \bar{\wp}(1,2)=(1 / 100) \leq$ $\psi(\bar{\wp}(2,0))=\psi(1 / 2)=(1 / 16)$.

Therefore, $\xi$ satisfies the conditions in Theorem 1, and hence it has a unique fixed point $g=1$.

Now, we present the following as an immediate consequence of Theorem 1.

Corollary 1. Let $(Y, \bar{\wp})$ be a complete controlled metric type space and $\xi: Y \longrightarrow Y$ be a mapping satisfying the following conditions:

(1) $\xi$ is continuous.

(2) There exists $\psi \in \Psi_{v}$ such that $\bar{\wp}(\xi g, \xi h) \leq \psi(\bar{\wp}(g, h))$, for all $g, h \in Y$.

Then, $\xi$ has a unique fixed point.

Proof. Define the function $\alpha: Y \times Y \longrightarrow[0,+\infty)$ via $\alpha(g, h)=1$. Note that $\xi$ is $\alpha$-admissible. Moreover, $\xi$ satisfies all the conditions of Theorem 1. So, $\xi$ has a unique fixed point.

In our next theorem, we replace the hypothesis of the continuity by a weaker condition.

Theorem 2. Let $(Y, \bar{\gamma})$ be a complete, controlled metric type space and $\xi: Y \longrightarrow Y$ be an $\alpha$ - $\psi$-contractive mapping for some $\psi \in \Psi_{s}$. Suppose that the following conditions hold:

(1) $\xi$ is $\alpha$-admissible.

(2) There exists $g_{0} \in Y$ such that $\alpha\left(g_{0}, \xi g_{0}\right) \geq 1$.

(3) If $\left\{g_{n}\right\}_{n=1}^{\infty}$ is a sequence in $Y$ such that $\alpha\left(g_{n}, g_{n+1}\right) \geq 1$ and $g_{n} \longrightarrow x$ as $n \longrightarrow \infty$, then $\alpha\left(g_{n}, g\right) \geq 1$ for all $n$.

Then, $\xi$ has a fixed point.

Proof. In proving the result, we follow the same steps as in the proof of Theorem 1 to construct a sequence $\left\{g_{n}\right\}_{n=1}^{\infty}$ that converges to a point $g \in Y$. The constructed sequence has the property $\alpha\left(g_{n}, g_{n+1}\right) \geq 1$, for all natural numbers $n$. The last assumption of the result implies that $\alpha\left(g_{n}, g\right) \geq 1$. We finally prove that $g$ is a fixed point for $\xi$. The triangle inequality implies that

$$
\begin{aligned}
\bar{\wp}(g, \xi g) & \leq \nu(g, \xi g)\left[\bar{\wp}\left(g, g_{n+1}\right)+\bar{\wp}\left(g_{n+1}, \xi g\right)\right] \\
& =v(g, \xi g) \bar{\gamma}\left(g, g_{n+1}\right)+\nu(g, \xi g) \bar{\gamma}\left(g_{n+1}, \xi g\right) .
\end{aligned}
$$

Note that the first term of the inequality, $\nu(g, \xi g) \bar{\wp}\left(g, g_{n+1}\right)$, converges to 0 , since $\left\{g_{n}\right\}$ converges to 0 . Also, the second term will be

$$
\begin{aligned}
\nu(g, \xi g) \bar{\wp}\left(g_{n+1}, \xi g\right) & \leq \nu(g, \xi g) \bar{\wp}\left(\xi_{g_{n}}, \xi g\right) \alpha\left(g_{n}, g\right) \\
& \leq \nu(g, \xi g) \psi\left(\bar{\wp}\left(g_{n}, g\right)\right) \\
& \leq \nu(g, \xi g) \bar{\wp}\left(g_{n}, g\right),
\end{aligned}
$$

which converges to 0 . Therefore, $\bar{\wp}(g, \xi g) \leq 0$, and hence $g$ is a fixed point for $\xi$.

\section{Conclusion}

Note that in Theorem 1, we used the continuity of the selfmapping on the controlled metric type space, which is a strong hypothesis. We want to bring to the reader's attention that we only used this hypothesis to prove that $\bar{\wp}(a, T a)=0$, and thus $a$ is a fixed point. So, the open question is can we omit the hypothesis of the continuity without replacing it with another weaker condition as in Theorem 2. In closing, we present the following open question:

Under what conditions we obtain the same results of Theorems 1 and 2 for self-mappings in double controlled metric type spaces [18].

\section{Data Availability}

No data were used to support this study.

\section{Conflicts of Interest}

The authors declare that they have no conflicts of interest.

\section{Acknowledgments}

The authors would like to thank Prince Sultan University for their support through NAMAM Research Group.

\section{References}

[1] S. Banach, "Sur les opérations dans les ensembles abstraits et leur application aux équations intégrales," Fundamenta Mathematicae, vol. 3, no. 1, pp. 133-181, 1922.

[2] N. Mlaiki, A. Mukheimer, Y. Rohen, N. Souayah, and T. Abdeljawad, "Fixed point theorems for $\alpha$ - $\psi$-contractive mapping in Sb-metric spaces," Journal of Mathematical Analysis, vol. 8, no. 5, pp. 40-46, 2017.

[3] A. Bakhtin, "The contraction mapping principle in almost metric spaces," Functional Analysis, vol. 30, pp. 26-37, 1989.

[4] S. Czerwik, "Contraction mappings in b-metric spaces," Acta Mathematica et Informatica Universitatis Ostraviensis, vol. 1, pp. 5-11, 1993.

[5] W. Shatanawi, K. Abodayeh, and A. Mukheimer, "Some fixed point theorems in extended b-metric spaces," UPB Scientific Bulletin, Series A, vol. 80, no. 4, 2018.

[6] H. Aydi, M. F. Bota, E. Karapinar, and S. Mitrovic, "A fixed point theorem for set-valued quasi-contractions in $b$-metric spaces," Fixed Point Theory and Applications, vol. 2012, p. 88, 2012.

[7] H. Aydi, M. F. Bota, E. Karapinar, and S. Moradi, "A common fixed point for weak phi-contractions on $b$-metric spaces," Fixed Point Theory, vol. 13, no. 2, pp. 337-346, 2012.

[8] T. Kamran, M. Samreen, and Q. Ul Ain, "A generalization of b-metric space and some fixed point theorems," Mathematics, vol. 5, no. 2, p. 19, 2017. 
[9] N. Mlaiki, H. Aydi, N. Souayah, and T. Abdeljawad, "Controlled metric type spaces and the related contraction principle," Mathematics, vol. 6, no. 194, 2018.

[10] K. Mehmet and H. Kiziltunc, "On some well-known fixed point theorems in b-metric spaces," Turkish Journal of Analysis and Number Theory, vol. 1, pp. 13-16, 2013.

[11] A. Mukheimer, " $\alpha-\psi$-contractive mappings on $b$-metric space," Journal Computational Analysis and Applications, vol. 18, no. 4, pp. 636-644, 2015.

[12] E. Ameer, H. Aydi, M. Arshad, H. Alsamir, and M. Noorani, "Hybrid multivalued type contraction mappings in $\alpha k$-complete partial b-metric spaces and applications," Symmetry, vol. 11, no. 1, p. 86, 2019.

[13] E. Karapinar, S. Czerwik, and H. Aydi, " $(\alpha, \psi)$-Meir-Keeler contraction mappings in generalized b-metric spaces," Journal of Function Spaces, vol. 2018, Article ID 3264620, 4 pages, 2018.

[14] H. Afshari, H. Aydi, and E. Karapınar, "On generalized $\alpha$ - $\psi$-Geraghty contractions on $b$-metric spaces," Georgian Mathematical Journal, vol. 27, no. 1, pp. 9-21, 2020.

[15] N. Mlaiki, N. Souayah, T. Abdeljawad, and H. Aydi, "A new extension to the controlled metric type spaces endowed with a graph," Advances in Difference Equations, vol. 2021, p. 94, 2021.

[16] M. U. Ali, H. Aydi, and M. Alansari, "New generalizations of set valued interpolative hardy-rogers type contractions in $b$ metric spaces," Journal of Function Spaces, vol. 2021, Article ID 6641342, 8 pages, 2021.

[17] E. Karapinar, A. Petrusel, and G. Petrusel, "On admissible hybrid geraghty contractions," Carpathian Journal of Mathematics, vol. 36, no. 3, pp. 433-442, 2020.

[18] T. Abdeljawad, N. Mlaiki, H. Aydi, and N. Souayah, "Double controlled metric type spaces and some fixed point results," Mathematics, vol. 6, no. 12, p. 320, 2018. 